\title{
SURGICAL MANAGEMENT OF DOG BITES IN A NEONATE
}

\author{
Luigino SANTECCHIA ${ }^{1}$, Fiammetta PIERSIGILLI², Barbara IACOBELLI ${ }^{2}$, Guglielmo \\ SALVATORI ${ }^{2}$, Cinzia AURITI ${ }^{*}$
}

${ }^{1}$ Plastic and Maxillofacial Surgery Unit Bambino Gesù Children's Hospital, Rome Italy, ${ }^{2}$ Department of Medical and Surgical Neonatology, Bambino Gesù Children's Hospital, Rome, Italy

\footnotetext{
*Corresponding author: cinzia.auriti@opbg.net Tel.: + 390668592427

Fax.: + 390668592832
}

Received: April 21, 2015

Accepted: September 22, 2015

Key words: Dog • Neonate - Dog bite.

\begin{abstract}
Objective - To describe a rare case of a very young female infant who suffered life threatening injuries by the family owned pet dog because she was ravaged at 10 days of life. Case report - The clinical conditions and vital signs of the infant on admission were stable. The buccal tissue presented multiple lacerations and the oral cavity was obstructed by multiple blood clots. Plastic surgery reconstruction was undertaken according to functional and aesthetic requirements. Antibiotic prophylaxis, tetanus vaccine and specific immunoglobulin were administered to the baby. Wound healing was excellent, despite contaminated wounds. Early and long term results of the intervention of reconstructive surgery are shown. We also review the literature to understand the background of this unexpected accident and its possible prevention. Conclusions - In children injuries by dog bites can be life-threatening, often imply aesthetic problems, as they involve mostly the neck and the head, and require medical and challenging surgical treatments. Despite the fact that infections do not occur frequently, in neonates, because of the lack of an efficient immune response, it is highly recommendable to start antibiotics and perform tetanus immunization together with the administration of specific immunoglobulins.
\end{abstract}

\section{Introduction}

Dogs are man's best friends.... but dogs bite more than 4 million people every year, even in developed countries and in the city environment. About one out of five of those who are bitten $(885,000$ people) require medical attention. What is more, children are more likely to suffer dog attacks. The majority of dog bites involve small children and are caused by a familiar dog (1).

According to the Center for Disease Control, dog bites of the facial region are most common in children (2). This usually results in severe acute trauma and has a significant long term psychological impact also due to the facial scars on the victims (3). Dog attacks on children are usually associated with significant "non-bite injuries" as well, such as head contusion, concussion, and abdominal and thoracic blunt trauma, which may be potentially dangerous (4).

We report a rare case of a very young female infant that was ravaged by a domestic dog at 10 days of life.

\section{Case report}

The dog (Alpenlaendische Dachsbracken) attacked the child while she was sleeping in her crib and the parents were in the adjoining room having dinner. The dog climbed over the crib, grabbed the baby by the right cheek, threw her on the ground and dragged her on the floor. The parents, attracted by the 


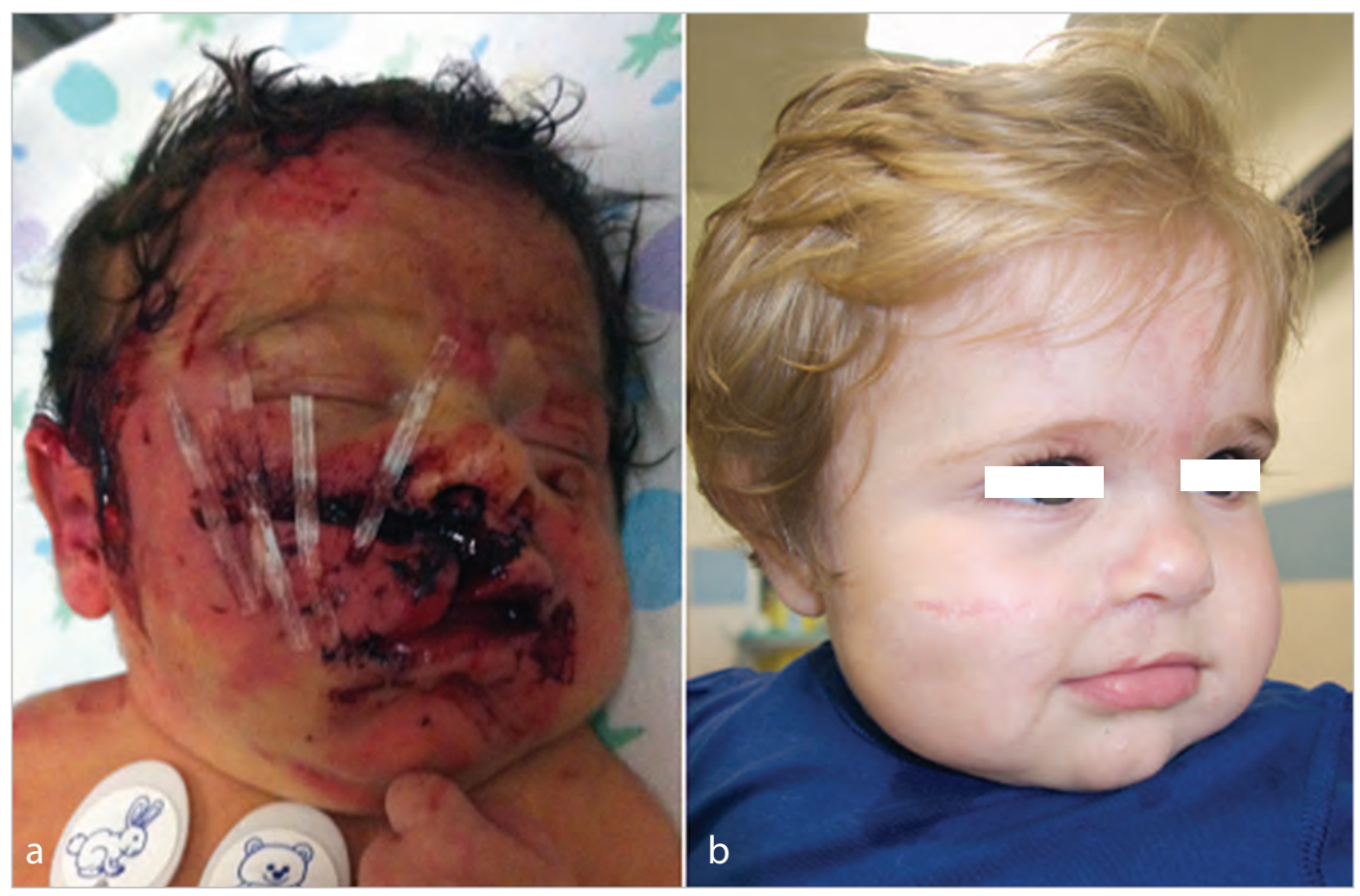

Fig. 1 Panel a: At admission the baby presented with copious bleeding, multiple lacerations and the oral cavity was obstructed by multiple blood clots; Panel $\boldsymbol{b}$ : facial mimic was restored at the age of 18 months.

crying of the baby, found her bleeding copiously and they brought her immediately to the emergency room. The clinical conditions and vital signs of the child on admission were surprisingly stable (100\% peripheral oxygen saturation on room air, normal blood pressure, crying) despite the copious bleeding and the obstruction of the oral cavity. In fact the buccal tissue presented multiple lacerations and the oral cavity was obstructed by multiple blood clots (Fig. 1, Panel a).

A computed tomography scan (CT) scan excluded both fractures of the skull, and intracranial hemorrhage. The bite lesions were severe, with cuts and grazes in the soft tissue, a $360^{\circ}$ section of the external ear canal and deep bite lesions in the area of the parotid gland and the right zygomatic-temporal region, along the course of the motor branch of the facial nerve. A large wound, including skin, mucosa and muscles, extended from the middle of the cheek to upper lip and right nasal ala. There was an extensive laceration of the nasal floor and destruction of the gingival mucosa fornix (Fig. 2, Panel a and Panel b).

The parotid gland was detached from the superficial muscular aponeurotic system with apparent stretching of the nerves emerging from the superficial anterior margin of the parotid, where the facial nerve exits and divides into distal motor branches: temporal, zygomatic, buccal, marginalis, and cervical, for the muscular structures of relevance, responsible for facial expression. The dental tooth germs of the upper gingival arch were damaged. The tongue was cut transversely into two parts, with excision of the two arteries and nerves, and copious bleeding. The slightest movement of the head resulted in the posterior portion of the tongue sliding back toward the glottis, with an immediate fall in saturation. Nasotracheal intubation was difficult due to the blood clots, tissue edema and oozing in the oropharynx. 


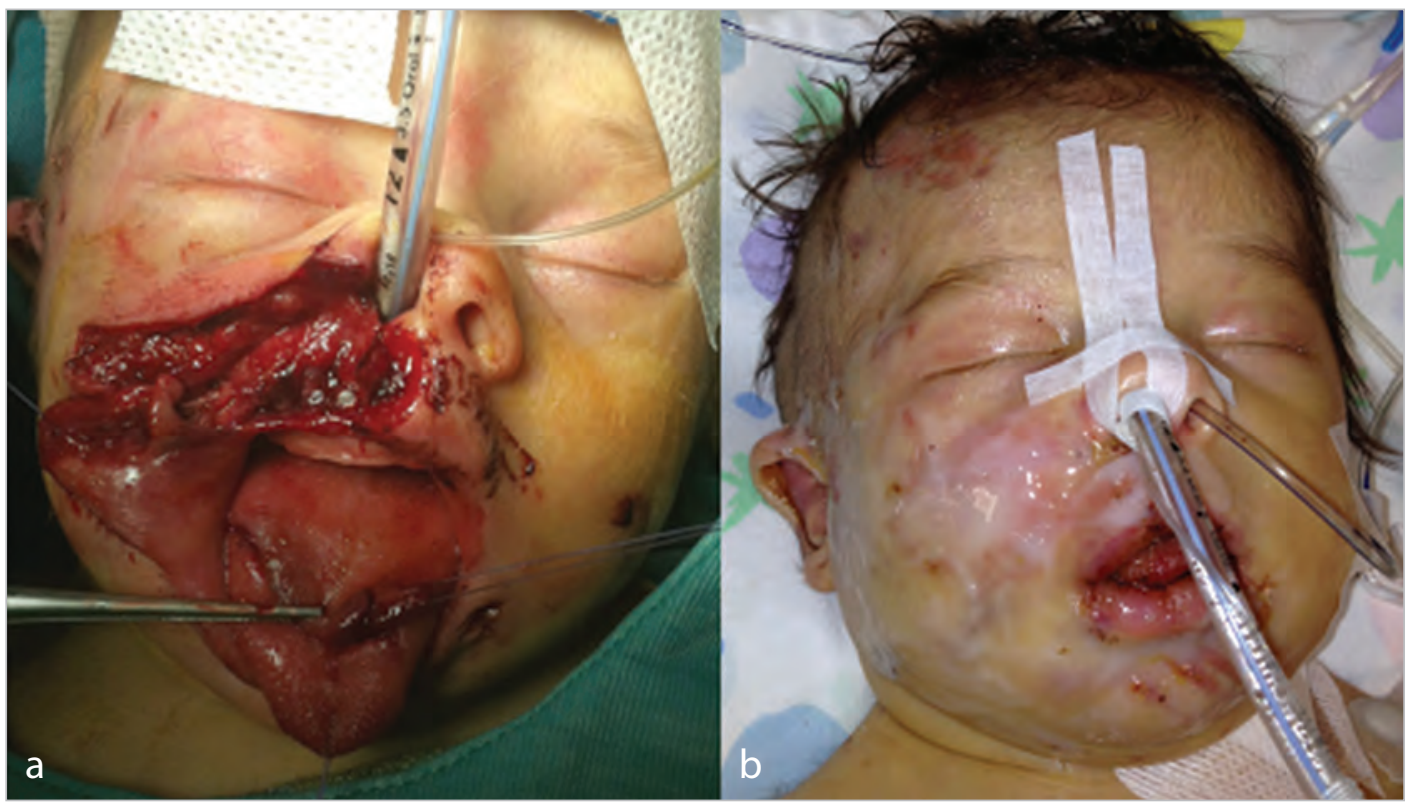

Fig. 2 Panel a: Prior to surgery the baby presented a large wound, comprising skin, mucosa, and muscles extending from the middle of the cheek to the upper lip and right nasal ala. The dental tooth germs of the upper gingival arch were damaged and the tongue was cut transversally. Panel $\boldsymbol{b}$ : Functional and aesthetic reconstruction was successfully performed with precision sutures obtained with loops for fine suturing. The loop consists of a suture thread in the shape of a flexible ring attached to a needle.

\section{Surgery}

Plastic surgical reconstruction was performed according to functional and aesthetic requirements, by repositioning layers of counterpart torn tissue, with precision sutures obtained with magnifying loops for fine suturing, after excision of clearly devitalized parts (Fig. 2, Panel b). The orbicularis oris muscle was sutured, the philtrum, the vermilion and the upper right vermillion border were reconstructed. The already difficult reconstruction of the nostril was further hampered by the presence of the nasal endotracheal tube, which induced artificial asymmetry. The right upper and lower left gingival alveolar arches were lacerated. Gems of deciduous teeth that were exposed and damaged were removed, to prevent damage to the buds of permanent teeth. The tongue was sutured, juxtaposing flaps and suturing muscles in layers with suture threads of synthetic polyglyconate Maxon 5-0 and 6-0. At the end of surgery the baby had twenty sutured wounds, some of which were full thickness, involving skin, muscle and mucosa.

\section{Medical therapy}

Due to the risk of infection, in the presence of a functionally immature immune system, broad spectrum antibiotics were started early before surgery and continued for one week after the intervention. Tetanus anatoxin and specific immunoglobulins against Tetanus were also administered early after admission to the Unit. Regarding rabies vaccine, the dog in question had been vaccinated, but was kept under observation. The baby did not receive a rabies vaccination.

\section{Follow up}

Fourteen days postoperatively the mouth appeared asymmetric due to right hemiparesis, (right lingual paresis associated with 
upper eyelid lagophthalmos and epiphora on the right), leading to transitory difficulties in feeding. At 18 months follow up facial mimic was fully recovered (Fig. 1, Panel b). The right hemiparesis and the lagophthalmos resolved, the nasal symmetry was satisfactory, and the long transverse scar on the right middle cheek was barely noticeable.

\section{Discussion}

In children, dog bites represent a serious problem, because they can be life-threatening and imply aesthetic problems, as they mostly involve the neck and the head (1). In our case the sliding back of the proximal part of the dissected tongue and the massive bleeding could have caused the infant to choke. We were afraid due to the significant loss of blood, which in this baby was probably not lethal because of the low blood pressure related to neonatal age.

The fact that the infant was crying likely prevented airway obstruction. Usually bite lesions are treated with juxtaposition of the torn edges, without suturing, to create natural drainage. We instead applied immediate suturing, after removal of clearly devitalized parts, due to massive surgical bleeding, but nevertheless no infection occurred.

The contamination of bite wounds is an important issue. Because of the presence of bacteria in the oral cavity, animal bite wounds are generally contaminated (5). The predominant pathogens in these wounds are the normal oral flora of the biting animal, along with human skin organisms and occasional secondary invaders (Staphylococcus aureus and Streptococcus pyogenes). Moreover, Pasteurella species are isolated from 50\% of dog bite wounds, and among anaerobes, $\mathrm{Bac}$ teroides species, fusobacteria, Porphyromonas species, Prevotella heparinolytica, proprionibacteria, and peptostreptococci are common anaerobes isolated from dog bite wounds (6).
Although infections occur infrequently in bitten children and adults, antibiotic therapy is indicated for all dog bites to the head and neck in neonates. The decision to administer oral or parenteral antibiotics depends on the depth and severity of the wound, and on the time since the bite occurred. In general children and adults should receive treatment with oral amoxicillin-clavulanate or with intravenous ampicillin-sulbactam or ertapenem, because agents such as dicloxacillin, cephalexin, erythromycin, and clindamycin have poor activity against Pasteurella multocida. Although cefuroxime, cefotaxime, and ceftriaxone are effective against Pasteurella multocida, they do not have good anaerobic spectra. Neonates can be treated with amoxicillin-clavulanate, ampicillin-sulbactam, and meropemen.

Our patient underwent antibiotic therapy early before surgery and until 1 week after the intervention, without adverse events. Furthermore, bite wounds are usually deep and contused, presenting a high risk of tetanus. This issue is particularly important in neonates who do not yet have a functionally efficient immune system. Tetanus immunisation and specific immunoglobulin should be routinely provided in neonatal bite wound management, without taking into account the immunisation status of the mother (7).

\section{Conclusions}

More than half of dog attacks are not provoked (8) and most dog bites occur in the home setting, by a dog known by the family (9). Children below the age of five are mostly involved (10). Most injuries occur when children are not properly supervised around familiar animals, in fact those bites that do occur are often preventable. The family dog can be a loving companion, but occasionally they can become aggressive without apparent reason, with or without provocation. There- 
fore children and especially neonates should be closely supervised even if they are at home with a familiar dog. Although a survey in 2008 suggested that dog bite rates for children had decreased compared to data from 1994, there still appears to be a need for effective prevention programs (3), including close supervision of children around pets and public education about responsible dog ownership. Ten commandments published in 1983 are still current (11); if the dog is to remain man's best friend, man must follow these simple rules: do not 1 . Hold your face close to a dog, 2. Allow dogs to roam unleashed, 3. Pet a strange dog, 4. Tease a dog, 5. Startle a dog, 6. Touch a sleeping dog, 7. Leave a small child and a dog alone, 8. Omit vaccination of a dog, 9. Leave a dog alone with strangers, and 10 . Ignore the warning signals of aggressive behavior.

Authors' contributions: Conception and design: LS, FP, CA; Acquisition, analysis and interpretation of data: LS, FP, CA, GS, BI; Drafting the article FP, CA; Revising it critically for important intellectual content: FP, CA.

Conflict of interest: The authors declare that they have no conflict of interest.

\section{References}

1. Abuabara A. A review of facial injuries due to dog bites. Med Oral Patol Oral Cir Bucal. 2006;11(4):E348-50.

2. Centers for Disease Control and Prevention (CDC). Nonfatal dog bite-related injuries treat- ed in hospital emergency departments--United States, 2001. MMWR Morb Mortal Wkly Rep. 2003;52(26):605-10.

3. Gilchrist J, Sacks JJ, White D, Kresnow MJ. Dog bites: still a problem? Inj Prev. 2008;14(5):296301.

4. Juang D, Sippey M, Zuckerbraun N, Rutkoski JD, Gaines BA. "Non-bite dog-related" injuries: an overlooked injury mechanism in the pediatric population. J Trauma. 2011;71(5 Suppl 2):S531-3.

5. Stevens DL, Bisno AL, Chambers HF, Everett ED, Dellinger P, Goldstein EJ,et al. Infectious Diseases Society of America. Practice guidelines for the diagnosis and management of skin and soft-tissue infections. Clin Infect Dis. 2005;41(10):1373-406.

6. Talan DA, Citron DM, Abrahamian FM, Moran GJ, Goldstein EJ. Bacteriologic analysis of infected dog and cat bites. Emergency Medicine Animal Bite Infection Study Group. N Engl J Med. 1999;340(2):85-92.

7. American Academy of Pediatrics. Red Book: Report of the Committee on Infectious Diseases 29th Edition: 708-709, 2012. [cited 2015 April 10]. Available from: http://www.amazon.com/Red-Book2012-Committee-Infectious/dp/158110703X.

8. Gandhi RR, Liebman MA, Stafford BL, Stafford PW. Dog bite injuries in children: a preliminary survey. Am Surg. 1999;65(9):863-4.

9. Brogan TV, Bratton SL, Dowd MD, Hegenbarth MA. Severe dog bites in children. Pediatrics. 1995;96(5 Pt 1):947-50.

10. Calkins CM, Bensard DD, Partrick DA, Karrer FM. Life-threatening dog attacks: a devastating combination of penetrating and blunt injuries. J Pediatr Surg. 2001;36(8):1115-7.

11. Wiseman NE, Chochinov H, Fraser V. Major dog attack injuries in children. J Pediatr Surg. 1983;18(5):533-6. 